\title{
Holography and the C-Theorem
}

\author{
Enrique Álvarez and César Gómez \\ Instituto de Física Teórica \\ Universidad Autónoma de Madrid \\ C-XVI, C.U. Cantoblanco \\ 28049 Madrid, Spain \\ E-mail: Enrigue AlvarezQuam
}

ABstract: We review the geometric definition of C-function in the context of field theories that admit a holographic gravity dual.

KEYwORDS: 'L̈olography, C-Theorem!

\section{Holographic C-Theorem}

\subsection{The Holographic Hypothesis}

The holographic hypothesis [1] $\left[\begin{array}{l}1 \\ 1\end{array}\right]$ postulates that the number of different quantum states in a given volume $V$ can not exceed $e^{\frac{A}{4 G_{D}}}$ where $A$ is the area of the corresponding boundary $\partial V$ and $G_{D}$ the Newton constant in $D$ space-time dimensions. This hypothesis is based on the idea that the maximun entropy associated with a volume $V$ is given by the Bekenstein-Hawking entropy $S=$ $\frac{A}{4 G_{D}}$. Following 't Hooft we can define the number of holographic degrees of freedom associated with a volume $V$ as

$$
N_{h d o f}=\frac{A}{4 \ln 2 G_{D}}
$$

On the other hand, Maldacena's conjecture [2, (3), 4 supersymmetric Yang Mills in flat Minkowski space-time, with gauge group $S U(N)$, and type IIB supergravity on $A d S_{5} \times S^{5}$ provided both the $A d S_{5}$ and the $S^{5}$ radii are given by

$$
R=l_{s}\left(g_{s} N\right)^{\frac{1}{4}}
$$

In order to check the holographic hypothesis for Maldacena's correspondence, we will start (following Susskind and Witten, [i]j] by considering $N=4$ supersymmetric Yang Mills in a box of topology $S^{3} \times \mathbb{R}$, where $\mathbb{R}$ represents time and the radius of the sphere $S^{3}$ is taken to be equal one. Let us now introduce an ultraviolet cutoff of size $\delta$. The number of degrees of freedom of the regularized theory would then be given by:

$$
N^{Y M}=\frac{N^{3}}{\delta^{3}}
$$

Next we move into the $A d S_{5}$ gravity dual. The metric of $A d S_{5}$ can be written as follows:

$$
d s^{2}=\frac{R^{2}}{\left(1-r^{2}\right)^{2}}\left(4 d X^{2}-\left(1+r^{2}\right)^{2} d t^{2}\right)
$$

In these coordinates the boundary is located at $r=1$. Notice that the holographic coordinate is now represented by $r$. We can introduce an infrared regulator by putting the regularized boundary at $r=1-\delta$. The area of the regularized boundary is given by

$$
A(\delta)=\frac{R^{3}}{\delta^{3}}
$$

Using the holographic hypothesis the number of holographic degrees of freedom associated with the volume inside the regularized boundary would be given by:

$$
N_{h d o f}=\frac{A(\delta)}{4 \ln 2 G_{5}}
$$

The quantum field theory ultraviolet cutoff will now be identified with the gravitational infrared cutoff.

By doing so we discover, using Maldacena's expression for the $A d S_{5}$ radius, that the number 
of holographic degrees of freedom associated with the volume with boundary at $r=1-\delta$ is precisely, up to a numerical factor $\frac{1}{4}$, the number of degrees of freedom of the regularized $N=4 \mathrm{su}-$ persymmetric Yang Mills theory, with ultraviolet cutoff equal $\delta$.

The main physical consequence of this result is to provide a solid basis for the interpretation of the holographic coordinate as a renormalization

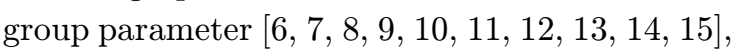
thus allowing to reinterpret Maldacena's correspondence in terms of the following simbolic relation: (Wilsonian QFT Renormalization group in $D$ space-time dimensions $)=($ Holographic principle in the gravity dual in $D+1$ space-time dimensions).

\subsection{Null Geodesic Congruences.}

Let us consider $A d S_{5}$ in horospheric (conformally flat) coordinates:

$$
d s^{2}=\frac{R^{2}}{z^{2}}\left(d z^{2}+d X^{2}\right)
$$

The boundary is now located at $z=0$. The first thing we need in order to check the holographic hypothesis in these coordinates is to define, in an intrinsic way, a 3-dimensional surface. This can be easily done using a congruence of null

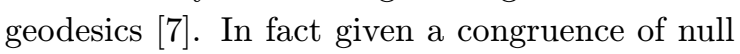
geodesics, in a space-time of dimension $D$, with tangent vector $k$, we can define a codimension two surface as follows. Let $p$ a point on the null geodesic and $T_{p}$ the tangent space at the point $p$. We define $V_{p}$ as the subspace of vectors in $T_{p}$ which are orthogonal to $k$. Now since $k$ is null we define the codimension two quotient space with respect to the equivalence relation $x-y \sim k$. This space will define the codimension two surface to be used later in order to define the holographic bound. Let us denote $h$ the induced metric on this surface. It is easy to prove [1] $\left.{ }_{1}\right]$ that

$$
\frac{d \sqrt{h}}{d \lambda}=\theta \cdot \sqrt{h}
$$

where $\lambda$ is the affine parameter of the null geodesic and $\theta$ the expansion of the null congruence.

Given a null geodesic $(X(\lambda), z(\lambda))$, it is easy to check that

$$
\sqrt{h}(\lambda)=\frac{R^{3}}{z^{3}(\lambda)}
$$

Using $z$ as the ultraviolet cutoff for the quantum field theory, we can the write the holographic hypothesis in more geometrical terms as

$$
\frac{\sqrt{h}}{G_{5}}=\frac{N^{2}}{z^{3}}
$$

The general idea of using null geodesic congruences in order to define a holographic area was

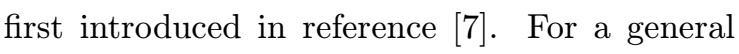
approach to holographic entropy based on null congruences see reference [1] 7 . In the appendix we present for completness the explicit construction of the codimension two volume element in the $A d S_{5}$ case using a different (although equivalent) approach.

\subsection{Holographic C- function}

Let us consider five dimensional space-time metrics preserving four dimensional Poincaré invariance:

$$
d s^{2}=a(r) d \vec{x}_{(1, d-1)}^{2}+d r^{2}
$$

where $d \vec{x}_{(1, d-1)}^{2}$ is the flat $d$-dimensional Minkowski metric.

Null geodesics are characterized by the null momentum:

$$
k^{\mu}=\frac{a_{(0)}}{a} k_{(0)}^{\mu}
$$

where $\mu, \nu \ldots=0 \ldots d-1$ and

$$
k^{d}=\left(\frac{a_{(0)}}{a}\right)^{1 / 2} k_{(0)}^{d}
$$

and the initial values are indeed null:

$$
a_{(0)} \eta_{\mu \nu} k_{(0)}^{\mu} k_{(0)}^{\nu}+\left(k_{(0)}^{d}\right)^{2}=0
$$

The expansion $\theta \equiv \nabla_{A} k^{A}(A, B \ldots=0, \ldots d)$ is given by:

$$
\theta=\frac{(1-d) k_{(0)}^{d} a^{\prime}}{2 a^{3 / 2}}
$$

(with $a^{\prime} \equiv \frac{d a}{d r}$ ), from which it stems that when $k_{(0)}^{d}>0$ as well as $a^{\prime}>0$, then $\theta<0$; that is, there is convergence of the null congruence.

Ulterior considerations will make use of the tangentially projected Ricci tensor:

$$
R_{A B} k^{A} k^{B}=\frac{3 a_{(0)}\left(k_{(0)}^{d}\right)^{2}\left(\left(a^{\prime}\right)^{2}-a a^{\prime \prime}\right)}{2 a^{3}}
$$


The null convergence condition, $R_{A B} k^{A} k^{B} \geq$ 0 then translates into

$$
\left(a^{\prime}\right)^{2} \geq a a^{\prime \prime}
$$

(This is marginally true in the AdS case, which is a maximally symmetric space so that $R_{A B} k^{A} k^{B}=$ 0 for a null vector $g_{A B} k^{A} k^{B}=0$ )

In conformally flat coordinates the metric (i1.î1) can be writen like

$$
d s^{2}=\Omega^{2}(z)\left(d X^{2}+d z^{2}\right)
$$

with $z$ the conformally flat coordinate defined by

$$
d r=\sqrt{a} d z
$$

Based on our previous discussion we suggest the following definition of holographic C-function

$$
C=\frac{\sqrt{h} \cdot z^{3}}{G_{5}}=\frac{z^{3} \Omega^{3}(z)}{G_{5}}
$$

with $\sqrt{h}$ given by

$$
\sqrt{h}=a(r)^{\frac{3}{2}}
$$

By construction this holographic C-function coincides, in the $A d S$ conformal case, with the value (in the large $N$ limit) of the central extension $N^{2}$ as defined by the Weyl anomaly. It is interesting to observe that the invariance of the C-function in the $A d S$ case is a direct consequence of the scale invariance of the metric with respect to dilatations: $X \rightarrow \lambda X, z \rightarrow \lambda z$.

In quantum field theory the C-function [i $\left.\overline{1}^{\prime} \overline{8}_{1}^{\prime}\right]\left[\begin{array}{l}1 \\ {[1}\end{array}\right.$ is a positive function of the couplings $g_{i}$ and on some length scale $L$ that satisfy the renormalization group equation, i.e independence on the renormalization group scale $\mu$

$$
\left(\mu \frac{\partial}{\partial \mu}+\beta_{i} \frac{\partial}{\partial g_{i}}\right) C\left(\mu L, g_{i}\right)=0
$$

The C-theorem is equivalent to the irreversibility of the renormalization group flow, more precisely

$$
L \frac{\partial}{\partial L} C\left(\mu L, g_{i}\right)<0
$$

Following our approach we will use as our scale $L$ the ultraviolet cutoff that we have identified with the conformally flat coordinate $z$. In that way we easily get the following relation for the geometric C-function

$$
z \frac{\partial}{\partial z} C=3 C\left(1+\frac{\Omega^{\prime}(z)}{\Omega(z)} z\right)
$$

Remembering now the value of the null congruence expansion $\theta_{A d S}=-\frac{1}{z}$ in AdS and defining $\theta_{\Omega}=\frac{\Omega^{\prime}(z)}{\Omega(z)}$ leads to

$$
z \frac{\partial}{\partial z} C=3 C\left(1-\frac{\theta_{\Omega}}{\theta_{A d S}}\right)
$$

This formula clearly expresses the fact that departure from conformal invariance is measured by the ratio $\frac{\theta_{\Omega}}{\theta_{A d S}}$.

\subsection{Jacobi Fields}

It is not difficult to show that any vector field which commutes with the tangent vector to a null geodesic congruence obeys the Jacobi equation

$$
\frac{D^{2}}{d \lambda^{2}} Z^{A}=-R_{B C D}^{A} Z^{C} k^{B} k^{D}
$$

The most general ${ }^{1}$ field $Z$ such that

$$
[Z, k]=0
$$

is

$$
Z^{A}=\frac{Z_{(0)}^{d}}{k_{(0)}^{d}} k^{A}+T^{A}
$$

where the components of $T$ read:

$$
T^{\mu}=Z_{(0)}^{\mu}-\frac{Z_{(0)}^{d}}{k_{(0)}^{d}} k_{(0)}^{\mu}
$$

and

$$
T^{d}=0
$$

Jacobi fields form a congruence of their own. Their expansion can be fully attributed to the component in the direction of $k$, and is given by:

$$
\theta_{J}=Z_{(0)}^{d} a_{(0)}^{1 / 2} \frac{(d-1) a^{\prime}}{a^{3 / 2}}
$$

It is interesting to notice that the only possible locus of convergence occurs at $a=\infty$

\footnotetext{
${ }^{1}$ Assuming only dependence on the holographic coordinate, $r$
} 


\subsection{Renormalization Group Flows}

The simplest example of a renormalization group flow, interpolating between two conformal field theory fixed points, is described, in the dual gravity picture, as a kink solution of the five dimensional $S U(4)$ gauged $N=8$ supergravity (see for instance, the references $\mathbb{R}_{1}^{9}, 100$ time metric of a kink solution preserving four dimensional Poincare invariance is of the type given in equation (i. $\overline{1} \overline{1}$ ). Based on the gravitational description of the conformal anomaly [ $[\overline{2} 0 \overline{0}]$ the following C-function was suggested in references $\left[\begin{array}{l}1 \\ 1\end{array}\right]$

$$
C=\frac{c t e}{A^{\prime 3}}
$$

with $e^{2 A}=a$ The monotonicity of this function with respect to the coordinate $r$ is guaranteed by

$$
A^{\prime \prime}<0
$$

Now, from the old equation (1, $\overline{1} \overline{1} \overline{0})$ we see that (i. dition, namely $R_{a, b} K^{a} K^{b}>0$ for any null vector $K$. It is interesting to notice that the appearance of the null convergence condition is already suggesting the holographic nature of the C-function in the sense discussed above. Namely the null convergence condition implies the focusing of the null congruence of geodesics used to define the codimension two surface employed to measure the number of holographic degrees of freedom. It is important to point out that both candidates for the C-function, namely the one defined in equation (1, $1 . \overline{3} \overline{2})$ based on the conformal anomaly and the geometric one defined in equation (1. $\left(1.20^{\prime}\right)$ coincide on the conformal points and both are monotonic if the null convergence condition is satisfied.

The C-function (1, $\overline{1} . \overline{3} \overline{2})$ can be written in more geometric terms as follows

$$
C=\frac{\sqrt{h}}{G_{5} \theta_{\Omega}^{3}}
$$

The difference with respect to the geometric definition ( $(1 \overline{2} \overline{2} \overline{0})$ is a difference -from the holographic point of view- in what is playing the role of ultraviolet quantum field theory cutoff, namely $z$ in one case or the analog of a Hubble length $\frac{1}{\theta_{\Omega}}$ in the other.
An alternative geometric description of the

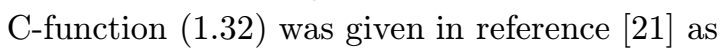

$$
C=\frac{c t e}{\sqrt{h} G_{5}|\theta|^{3}}
$$

$\theta$ being the null geodesic expansion.

\subsection{Raychaudhuri's Equation and Confor- mal Invariance}

Let us now consider a box in euclidean $\mathbb{R}^{3}$ of side length equal $L$. We are interested in the behaviour of the volume of the box with respect to dilatations $L \rightarrow \lambda L$. Obviously we get

$$
\frac{d \lambda^{3} L^{3}}{d \lambda}=3 \lambda^{2} L^{3}
$$

We can define the expansion coefficient $\theta=\frac{3}{\lambda}$. This expansion trivially satisfies the equation:

$$
\frac{d \theta}{d \lambda}=-\frac{\theta^{2}}{3}
$$

This is Raychaudhuri's equation for the null congruence expansion in $A d S_{5}$. This simple geometrical fact is the reason for a constant $\mathrm{C}$ - function ( i.e conformal invariance ) in $A d S_{5}$. Namely the scaling of null congruences is the same of that of three dimensional euclidean volume. Any departure of conformal invariance is defined by adding the effect of "holographic" matter fields in Raychaudhuri's equation i.e adding the term $-R_{a b} K^{a} K^{b}$.

Thus from the holographic point of view the variation of the $\mathrm{C}$-function measures the diffrence in the scaling behaviour of three dimensional euclidean volume and the three dimensional volume associated with a congruence of null geodesics.

\section{Appendix}

\section{Conformally Invariant case}

Let us employ the coordinates introduced by Fefferman and Graham ([22] ), and used in ([20 reproduce the Weyl anomaly which enjoy the property that the boundary lies at $\rho=0$ :

$$
d s^{2}=\frac{1}{\rho} g_{\mu \nu}(x, \rho) d x^{\mu} d x^{\nu}+\frac{l^{2}}{4 \rho^{2}} d \rho^{2}
$$

(where $l$ is a length scale associated to the total curvature of the spacetime, what we called 
before the radius, $R$ ). We shall denote by $x^{A}$ the set of the five coordinates, $x^{\mu}, \rho$, where greek letters run from $0 \ldots 3$ and capital latin letters from $0 \ldots 4$, with $x^{4} \equiv \rho$. We shall furthermore concentrate in the simplest case in which

$$
g_{\mu \nu}=\eta_{\mu \nu}
$$

It is not difficult to compute the null geodesics in the preceding manifold. They are given by:

$$
\begin{gathered}
x^{\mu}=\frac{l^{4} \dot{x}_{(0)}^{\mu} \dot{\rho}_{(0)}^{2}}{2 \dot{x}_{(0)}^{2}\left(l^{2} \dot{\rho}_{(0)}-2 \dot{x}_{(0)}^{2}\left(\lambda-\lambda_{(0)}\right)\right.}+ \\
x_{(0)}^{\mu}-\frac{l^{2} \dot{\rho}_{(0)} \dot{x}_{(0)}^{\mu}}{2 \dot{x}_{(0)}^{2}} \\
\rho=\frac{l^{6} \dot{\rho}_{(0)}^{4}}{4 \dot{x}_{(0)}^{2}\left(l^{2} \dot{\rho}_{(0)}-2 \dot{x}_{(0)}^{2}\left(\lambda-\lambda_{(0)}\right)\right)^{2}}
\end{gathered}
$$

There are two different behaviours depending on the sign of $\dot{\rho}_{(0)}$ : if $\dot{\rho}_{(0)}>o$, the geodesic starts at $\left(x_{(0)}^{\mu}, \rho_{(0)}\right)$ at $\lambda=\lambda_{(0)}$, and reaches infinity at a finite value of the affine parameter $\lambda_{c}$. These geodesics can be thought of as starting at the boundary $\rho=0$ at $\lambda=-\infty$.

When $\dot{\rho}_{(0)}<0$, instead, the geodesic again starts at $\left(x_{(0)}^{\mu}, \rho_{(0)}\right)$ at $\lambda=\lambda_{(0)}$, but now reaches the boundary $\mathcal{I}$ at $\lambda=+\infty$. They can be thought of as coming from the infinity at $\lambda=-\lambda_{c}$.

The tangent vector is given by:

$$
\begin{aligned}
& k^{\mu}=\dot{x}_{0}^{\mu} \rho / \rho_{(0)} \\
& k^{4}=\dot{\rho}_{(0)} \rho^{3 / 2} \rho_{(0)}^{-3 / 2}
\end{aligned}
$$

(where the timelike vector $\dot{x}_{0}^{\mu}$ is normalized through $\left.\eta_{\mu \nu} \dot{x}_{0}^{\mu} \dot{x}_{0}^{\nu}=-\frac{l^{2} \dot{\rho}_{(0)}^{2}}{4 \rho_{(0)}}\right)$.

The optical scalars are easily determined from:

$$
\begin{aligned}
\nabla_{\mu} k_{\nu} & =-\frac{1}{2} \dot{\rho}_{(0)} \eta_{\mu \nu}(\rho)^{-1 / 2} \rho_{(0)}^{-3 / 2} \\
\nabla_{\mu} k_{4} & =\frac{\eta_{\mu \nu} \dot{x}_{(0)}^{\nu}}{2 \rho \rho_{(0)}} \\
\nabla_{4} k_{4} & =\nabla_{4} k_{\mu}=\frac{\dot{\rho}_{(0)} l^{2}}{8}\left(\rho \rho_{(0)}\right)^{-3 / 2}
\end{aligned}
$$

The fact that this tensor is symmetric means that the congruence is irrotational $\omega=0$, which in turn conveys the fact that it is hypersurface orthogonal. The expansion,

$$
\theta \equiv \nabla_{A} k^{A}=-3 / 2 \dot{\rho}_{(0)} \rho^{1 / 2} \rho_{(0)}^{-3 / 2}
$$

This physically means that there is gravitational focusing towards Penrose's boundary, $\mathcal{I}$.

What we need now is to determine an area which is naturally associated to the null congruence. The normal hypersurface suffers from the ambiguity that the vector $k$ itself belongs to it. Although it is possible to perform an analysis along these lines (as it is done in Hawking and Ellis's book), there is a physically more transparent construction, to which we turn.

We shall introduce a sort of Newman-Penrose fünfbein (except that, living in an odd unmber of dimensions, complex techniques are not useful). For that purpose, we shall first consider another null vector, $l^{A} \partial_{A}$, such that $g_{A B} l^{A} k^{B}=-1$, and it could be propagated in a parallel way along the null geodesic, i.e., $k^{C} \nabla_{C} l^{A}=0$. It turns out that this construction defines a codimension two hypersurface and besides, that the Lie derivative of the logarithm of the induced volume element is directly related to the null congruence expansion $\theta$.

The most general vector $l^{A}$ obeying the requeriments as above is given by:

$$
\begin{aligned}
l^{\mu}= & \left(l_{(0)}^{\mu}-\left(l_{(0)}^{4}+\frac{2 \rho_{(0)}^{2}}{l^{2} \dot{\rho}_{(0)}}\right) \frac{\dot{x}_{(0)}^{\mu}}{\dot{\rho}_{(0)}}\right)\left(\rho / \rho_{(0)}\right)^{1 / 2}+ \\
& \frac{l_{(0)}^{4} \rho \dot{x}_{(0)}^{\mu}}{\dot{\rho}_{(0)} \rho_{(0)}}+\frac{2 \dot{x}_{(0)}^{\mu} \rho_{(0)}^{2}}{l^{2} \dot{\rho}_{(0)}^{2}} \\
l^{4}= & l_{(0)}^{4}\left(\rho / \rho_{(0)}\right)^{3 / 2}-\frac{2 \rho_{(0)}\left(\rho \rho_{(0)}\right)^{1 / 2}}{\dot{\rho}_{(0)} l^{2}}
\end{aligned}
$$

where $\eta_{\mu \nu} k_{(0)}^{\mu} l_{(0)}^{\nu}=l_{(0)}^{4}-c \eta_{\mu \nu} l_{(0)}^{\mu} l_{(0)}^{\nu}=0$

Let us choose the simplest possibility (while keeping all the generality in $k^{A}$ ), namely

$$
l^{A}=\frac{2 \rho_{(0)}}{\dot{\rho}_{(0)} l^{2}}\left(\frac{1}{2} k_{(0)}^{\mu},-\left(\rho_{(0)} \rho\right)^{1 / 2}\right)
$$

It is now a simnple matter to show that the three spacelike vectors $e_{i}^{A}$ orthogonal to both $k^{A}$ and $l^{A}$ are given by:

$$
e_{(i)}^{A}=\left(e_{(i)}^{\mu}, 0\right)
$$

where $\eta_{\mu \nu} e_{(i)}^{\mu} e_{(i)}^{\nu}=\rho$ and $\eta_{\mu \nu} e_{(i)}^{\mu} k_{(0)}^{\nu}=0$. This in turn yields the $\rho$ dependence, namely $e_{(i)}^{\mu}=$ $\rho^{1 / 2} E_{(i)}^{\mu}$. where $\eta_{\mu \nu} E_{(i)}^{\mu} E_{(i)}^{\nu}=1$ and $\eta_{\mu \nu} E_{(i)}^{\mu} k_{(0)}^{\nu}=$ 0 
The finite equations of the hypersurface (which lies on a slice of constant $\rho$ ) are then given by:

$$
x^{\mu}=E_{(i)}^{\mu} \xi^{i}
$$

Correspondingly, the induced metric is:

$$
d s^{2}=\frac{1}{\rho} \delta_{i j} d \xi^{i} d \xi^{j}
$$

and the volume element ${ }^{2}$ scales as

$$
A_{d-2} \sim \sqrt{h} \sim \rho^{-3 / 2}
$$

in such a way that indeed

$$
\begin{aligned}
£(k) A_{d-2} & \equiv k^{A} \nabla_{A} A_{d-2} \\
& =-\frac{3 \dot{\rho}_{(0)} \rho^{1 / 2}}{2 \rho_{(0)}^{3 / 2}} A_{d-2} \equiv \theta A_{d-2}
\end{aligned}
$$

\section{A non conformal case}

The following metric was introduced in [203].

$$
d s^{2}=\frac{1}{\rho} \eta_{\mu \nu} d x^{\mu} d x^{\nu}+\frac{l_{c}^{2}}{\rho^{4}} d \rho^{2}
$$

Here $l_{c}$ sets the scale of the curvature, although in the present case the latter is of course not constant. Please note the striking similarity with AdS in the coordinates just employed; the difference stemming from the power of the holographic coordinate in the last term. We shall actually employ the coordinate $g \equiv \frac{1}{\rho}$, so that

$$
d s^{2}=g d \vec{x}_{4}^{2}+l_{c}^{2} d g^{2}
$$

The general formulas for Poincaré invariant metrics yield for the tangent vectors to null geodesics the expression:

$$
\begin{aligned}
& k_{\mu}=c_{\mu} \\
& k_{4}=\frac{c^{0}}{\gamma} g^{-1 / 2}
\end{aligned}
$$

with $\eta_{\mu \nu} c^{\mu} c^{\nu}=-\frac{\left(c^{0}\right)^{2}}{\gamma}$. corresponding to the parametrized null geodesics:

$$
\begin{aligned}
& x^{\mu}=x_{(0)}+\frac{2 c^{\mu} \gamma}{c^{0}}\left(\frac{3 c^{0}}{2 \gamma}\left(\lambda-\lambda_{0}\right)+g_{(0)}^{3 / 2}\right)^{1 / 3} \\
& g=\left(\frac{3 c^{0}}{2 \gamma}\left(\lambda-\lambda_{0}\right)+g_{(0)}^{3 / 2}\right)^{2 / 3}
\end{aligned}
$$

\footnotetext{
${ }^{2}$ The hypersurfaces are non compact in general, so that their volume is infinite. We shall mostly be interested in the volume density instead, which is a meaningful (and finite) quantity.
}

It is plain that, given any point with coordinates $x_{(0)}^{\mu}, g_{(0)}$, there is a null geodesic starting at it. This means, in mathematical terms, that there is a null geodesic congruence. The vanishing of the rotation conveys the fact that they are all normal to the codimension one hypersurface

$$
c_{\mu} x^{\mu}+2 \frac{c^{0}}{\gamma} g^{1 / 2}=\text { constant }
$$

Given a curve

$$
\begin{aligned}
& x_{(0)}^{\mu}=x_{(0)}^{\mu}(\lambda) \\
& g_{(0)}=g_{(0)}(\lambda)
\end{aligned}
$$

the connecting vector is given by:

$$
\begin{aligned}
Z^{\mu} & =\frac{\gamma \beta^{\mu}}{g} \dot{g_{(0)}} g_{(0)}^{1 / 2}+\dot{x}_{(0)}^{\mu} \\
Z^{4} & =g_{(0)}^{1 / 2} \dot{g}_{(0)} g^{-1 / 2}
\end{aligned}
$$

This vector conmutes with $k^{A}$, and indeed

$$
Z=\frac{\gamma \dot{g}_{(0)} g_{(0)}^{1 / 2}}{c^{0}} k+\dot{x}_{(0)}^{\mu} \partial_{\mu}
$$

We want now to make explicit the construction of the volume element of codimension 2 associated to the null congruence in this case. The most general null vector $l^{A}$, normalized in such a way that

$$
k . l=-1
$$

is

$$
l=l^{0}(1, \vec{n} \sin \chi, g \cos \chi)
$$

where $l^{0} \equiv\left(c^{0}\left(1-\frac{g^{1 / 2} \cos \chi}{\gamma}\right)-\vec{c} \cdot \vec{n} \sin \chi\right)^{-1}$. The vector $\vec{n}$ is a three-dimensional unit vector, $\vec{n}^{2}=$ 1 , and $\chi$ is an arbitrary angle.

The spacelike 3 -surface orthogonal to both $k$ and $l$ will be given, in terms of the parameters $g_{i}$, by:

$$
\frac{\partial \Phi^{A}}{\partial g^{i}} g_{A B} k^{B}=\frac{\partial \Phi^{A}}{\partial g^{i}} g_{A B} l^{B}=0
$$

Whose general solution is:

$$
\begin{aligned}
t & =t_{(0)}+\vec{v} \cdot \vec{n} \sin \chi+g_{3} \cos \chi \\
\vec{x} & =-t_{(0)} \vec{n} \sin \chi+\vec{v} \\
g & ==-t_{(0)} \cos \chi+g_{3}
\end{aligned}
$$


where the vector $\vec{v}$ is determined in terms of the parameters $g_{i}$ through:

$$
\begin{aligned}
v_{1}= & g_{1}+\left[V_{(0)}-t_{(0)} \vec{\beta} \cdot \vec{n} \sin \chi-g_{3} \cos \chi+\right. \\
& \left.\frac{2}{\gamma} \sqrt{g_{3}-t_{(0)} \cos \chi}\right] \frac{n_{1} \sin \chi-\beta_{1}}{\sin ^{2} \chi+\beta^{2}-2 \vec{n} \cdot \vec{\beta} \sin \chi} \\
v_{2}= & g_{2}+\left[V_{(0)}-t_{(0)} \vec{\beta} \cdot \vec{n} \sin \chi-g_{3} \cos \chi+\right. \\
& \left.\frac{2}{\gamma} \sqrt{g_{3}-t_{(0)} \cos \chi}\right] \frac{n_{2} \sin \chi-\beta_{2}}{\sin ^{2} \chi+\beta^{2}-2 \vec{n} \cdot \vec{\beta} \sin \chi} \\
v_{3}= & \frac{g_{1}\left(n_{1} \sin \chi-\beta_{1}\right)+g_{2}\left(n_{2} \sin \chi-\beta_{2}\right)}{\beta_{3}-n_{3} \sin \chi} \\
& +\left[V_{(0)}-t_{(0)} \vec{\beta} \cdot \vec{n} \sin \chi-g_{3} \cos \chi+\right. \\
& \left.\frac{2}{\gamma} \sqrt{g_{3}-t_{(0)} \cos \chi}\right] \\
& \frac{n_{3} \sin \chi-\beta_{3}}{\sin ^{2} \chi+\beta^{2}-2 \vec{n} \cdot \vec{\beta} \sin \chi}
\end{aligned}
$$

Not all the vectors $\frac{\partial \Phi^{A}}{\partial g_{i}}$ do parallel propagate along the null geodesics. It is easy to check that an arbitrary vector $v_{(0)}^{A}$ defined at a particular point $g_{(0)}$ propagates in a parallel way provided its $g$ dependence is fixed to be:

$$
\left(v_{(0)}^{\mu} \frac{g_{(0)}^{1 / 2}}{g^{1 / 2}}+\gamma v_{(0)}^{4} \beta^{\mu}\left(\frac{g_{(0)}^{1 / 2}}{g}-\frac{1}{g^{1 / 2}}\right), v_{(0)}^{4} \frac{g_{(0)}^{1 / 2}}{g^{1 / 2}}\right)
$$

This can be shown to imply the necessary condition for the fünfbein to be parallel transported, namely

$$
\vec{\beta}=\vec{n} \sin \chi
$$

(i.e., only when these conditions are fulfilled, can the fünfbein be parallel transported) which in turn singles out the associated spacelike normal hypersurface as lying in a slice of constant $g$ and, besides

$$
t=\vec{x} \cdot \vec{n} \sin \chi+\text { constant }
$$

The induced metric on the hypersurface is then given simply by:

$$
d s^{2}=g\left(\delta_{i j}-n_{i} n_{j}\right) d x^{i} d x^{j}
$$

in such a way that the volume density reads

$$
A_{d-2} \sim g^{3 / 2} \cos \chi
$$

We are now ready to recover in a very explicit way the interpretation of the expansion as the logarithmic derivative of the natural $d-2$ volume element associated to the null congruence:

$$
k^{A} \nabla_{A} A_{d-2}=\frac{3 c^{0}}{2 \gamma} g^{-3 / 2} A_{d-2}=\theta A_{d-2}
$$

\section{Acknowledgments}

This work has been supported by the European Union TMR programs FMRX-CT96-0012 Integrability, Non-perturbative Effects, and Symmetry in Quantum Field Theory and ERBFMRXCT96-0090 Beyond the Standard model as well as by the Spanish grants AEN96-1655 and AEN961664 .

\section{References}

[1] 't Hooft,Dimensional reduction in Quantum Gravity gr-qc/9310026 L.Susskind, The world as a hologram hep-th/9409089

[2] J. Maldacena, The large $N$ limit of superconformal field theories and supergravity, Adv.Theor.Math.Phys.2:231-252,1998, hep-th/9711200.

[3] E. Witten, Anti de Sitter Space and Holography, hep-th/9802150.

[4] S. S. Gubser, I. R. Klebanov and A. M. Polyakov, Gauge theory correlators from non-critical string theory, Phys. Lett. B428 (1998) 105 hep-th/9802109

[5] L. Susskind and E. Witten, The Holomorphic Bound in AdS-Spaces, hep-th/9805114.

[6] E.T.Akhmedov, A Remark on the AdS/CFT correspondence and the Renormalization Group Flow, hep-th/9906217,Phys.Lett. B442 (1998) 152.

[7] E. Álvarez and C. Gómez, Geometric Holography, the Renormalization Group and the c-Theorem, hep-th/9807226,Nucl.Phys. B541 (1999) 441-460.

[8] A. W. Peet and J. Polchinski, UV/IR relations in AdS dynamics, Phys. Rev. D59 (1999) 065011 hep-th/9809022.

[9] L. Girardello, M. Petrini, M. Porrati and A. Zaffaroni, Novel local CFT and exact results on perturbations of $N=4$ super YangMills from AdS dynamics JHEP 9812 (1998) 022 hep-th/9810126.

[10] J. Distler and F. Zamora, Non-supersymmetric conformal field theories from stable anti-de Sitter spaces, Adv. Theor. Math. Phys. 2 (1999) 1405 hep-th/9810206.

[11] V. Balasubramanian and P. Kraus, Spacetime and the Holographic Renormalization Group, hep-th/9903190. 
[12] D. Z. Freedman, S. S. Gubser, K. Pilch and N. P. Warner, Renormalization group flows from holography supersymmetry and a c-theorem, hep-th/9904017

[13] H. Verlinde, Holography and compactification, Nucl. Phys. B580 (2000) 264 hep-th/9906182.

[14] J. de Boer, E. Verlinde and H. Verlinde, On the holographic renormalization group, JHEP 0008 (2000) 003 hep-th/9912012

[15] E. Verlinde and H. Verlinde, RG-flow, gravity and the cosmological constant, JHEP 0005 (2000) 034 hep-th/9912018.

[16] S.W. Hawking and G.F.R. Ellis, Singularities in homogeneous world models, Phys. Lett. 17 (1965) 246.

The Large Scale Structure of Spacetime, Cambridge University Press, 1973.

[17] R. Bousso, Holography in general space-times, JHEP 9906 (1999) 028 hep-th/9906022.

[18] A.B. Zamolodchikov, JETP Lett. 43 (1986),730.

[19] J. L. Cardy, Is There A C Theorem In FourDimensions?, Phys. Lett. B215 (1988) 749.

[20] M. Henningson and K. Skenderis, The holographic Weyl anomaly, JHEP 9807 (1998) 023 hep-th/9806087.

[21] V. Sahakian, Holography, a covariant c-function and the geometry of the renormalization group, hep-th/9910099.

[22] C. Fefferman and R. Graham, Conformal Invariants,in Elie Cartan et les mathématiques d'aujourdhui, Asterisque,(1985) 95.

[23] E. Alvarez and C. Gomez, The confining string from the soft dilaton theorem, Nucl. Phys. B566 (2000) 363 hep-th/9907158. 